\title{
Clinical application of nano-carbon to improve the accuracy of lymph node staging in patients with advanced gastric cancer receiving neoadjuvant chemotherapy: a prospective randomized controlled trial
}

\author{
Peigang Yang^, Yuan Tian, Bibo Tan, Pingan Ding, Honghai Guo, Yang Liu, Zhidong Zhang, Yong Li, \\ Qun Zhao
}

Department of Surgery, the Fourth Hospital of Hebei Medical University, Shijiazhuang, China

Contributions: (I) Conception and design: Q Zhao; (II) Administrative support: Y Li, B Tan; (III) Provision of study materials or patients: Z Zhang, P Yang; (IV) Collection and assembly of data: Y Tian, P Ding; (V) Data analysis and interpretation: P Yang, H Guo, Y Liu; (VI) Manuscript writing: All authors; (VII) Final approval of manuscript: All authors.

Correspondence to: Qun Zhao. Department of Surgery, the Fourth Hospital of Hebei Medical University, Shijiazhuang 050011, China.

Email: zhaoqun@hebmu.edu.cn.

Background: The significance of nano-carbon for lymph node staging in radical gastrectomy for gastric cancer (GC) has been confirmed, but studies on its application for GC patients treated with neoadjuvant chemotherapy (NCT) are rare. The purpose of this study was to explore the clinical value of using carbon nanoparticles suspension injections (CNS) to improve the accuracy of lymph node staging ( $\mathrm{N}$ staging) of NCT for advanced GC.

Methods: 160 advanced GC patients receiving preoperative NCT were enrolled, according to the random number generated by computer, the enrolled patients were randomly divided into two groups: experimental group ( $n=80)$ and control group $(n=80)$. The experimental group received endoscopic injection of CNS within 24 hours prior to NCT, while the control group received this within 24 hours post NCT and before D2 radical resection. SOX [oxaliplatin: $130 \mathrm{mg} /$ (body surface area, BSA): $\mathrm{m}^{2}$, first day + S-1: (BSA: $<1.25 \mathrm{~m}^{2}, 40 \mathrm{mg}$ each time; $\geq 1.25$ to $<1.5 \mathrm{~m}^{2}, 50 \mathrm{mg}$ each time; $\geq 1.5 \mathrm{~m}^{2}, 60 \mathrm{mg}$ each time), 2 times a day, for 2 weeks] was chosen as the NCT regimen, repeat every 3 weeks, 4 cycles were performed preoperative. Surgery was performed 3 weeks after the end of the 4 cycles of chemotherapy. The staining rate, metastasis rate, metastasis rate of stained lymph nodes, postoperative complication rate, and $\mathrm{N}$ staging of the two groups were analyzed and compared.

Results: A total of 3,197 lymph nodes were harvested in the experimental group, including 384 metastatic lymph nodes, 1,424 stained lymph nodes, and 210 metastatic stained lymph nodes. The total number of lymph nodes harvested in the control group was 2,565, including 244 metastatic lymph nodes, 796 stained lymph nodes, and 94 metastatic stained lymph nodes. Compared with the control group, a higher rate of stained lymph nodes, a higher total number of lymph nodes, and an increased number of metastatic lymph nodes were detected in the experimental group.

Conclusions: The application of CNS before NCT in patients with advanced GC can minimize lymph node staging bias after NCT and improve its accuracy.

Trial Registration: Chinese Clinical Trial Registry ChiCTR2100047407.

Keywords: Carbon nanoparticles; neoadjuvant chemotherapy (NCT); advanced gastric cancer (advanced GC); lymph node staging (N staging)

Submitted Jul 07, 2021. Accepted for publication Sep 27, 2021.

doi: 10.21037/jgo-21-457

View this article at: https://dx.doi.org/10.21037/jgo-21-457

^ ORCID: 0000-0002-1055-4125. 


\section{Introduction}

Surgery remains an important method for treating advanced gastric cancer (GC), including complete tumor resection, enough cut edge, and regional lymphadenectomy. Among these, the number of dissected lymph nodes is an important index to test the quality of radical gastrectomy and is an independent risk factor affecting the prognosis of patients. Studies have shown that to more accurately evaluate the prognosis of advanced GC patients, the number of lymph nodes detected should be more than 30 (1-3).

Currently, neoadjuvant chemotherapy (NCT) has become the recommended treatment for advanced GC because it effectively increases the surgical radical degree and reduces the postoperative recurrence rate (4-7). However, tumor regression reaction after NCT can lead to a decrease in the number of lymph nodes and reduction in their volume. In turn, this may lead to a reduction in the number of lymph nodes detected and the migration of lymph node staging (N staging) after NCT, which affects the surgical quality, the evaluation of chemotherapy efficacy, and the judgment of prognosis (8). The studies on how to improve the $\mathrm{N}$ staging accuracy after neoadjuvant therapy for gastric cancer are rare.

Carbon nanoparticles with a diameter of $150 \mathrm{~nm}$, after injected into submucosal tissue of the tumour, carbon nanoparticles can actively transported or passively transported to the local lymphatic vessels and lymph nodes are black stained, so lymph nodes can be easily identified and obtained, and once the lymph nodes are stained, they will not fade due to factors such as chemotherapy. As a result, the number of lymph nodes detected increased after GC surgery, and can reduce the deviation of postoperative pathological $\mathrm{N}$ staging. In previous studies on the application of carbon nanoparticles suspension injections (CNS) in NCT for advanced GC, CNS injections were carried out before or during the operation after the completion of NCT. However, reactions to chemotherapy could cause lymphoid tissue fibrosis, and regional lymph nodes were difficult to adequately stain within a limited time, affecting their detection rate (9). Therefore, how to effectively solve the problem of reduced lymph node detection caused by NCT and improving the accuracy of N staging has important clinical significance.

The purpose of this study was to investigate the clinical value of CNS in improving the accuracy of lymph node staging in patients with advanced GC by injecting CNS before and after NCT, and further analyzing the relevant data collected. We present the following article in accordance with the CONSORT reporting checklist (available at https://dx.doi.org/10.21037/jgo-21-457).

\section{Methods}

\section{Patient selection criteria}

This study was designed as a randomized, parallel controlled clinical trial. A total of 160 patients with advanced GC who planned to receive NCT in the Fourth Hospital of Hebei Medical University from January 2020 to January 2021 were recruited. Using a random number table and allocation ratio is $1: 1$, patients were divided into an experimental group $(\mathrm{n}=80)$ and a control group $(\mathrm{n}=80)$. All patients corresponded to the following inclusion criteria: (I) 18-70 years old; (II) pathologically diagnosed as GC by gastroscopy and biopsy; and (III) abdominal computer tomography (CT) combined with laparoscopic exploration assessed the clinical stage as advanced T3-4N+M0. Patients were assessed before cancer treatment to confirm their ability to tolerate NCT and surgery by the treatment team.

The exclusion criteria included any of the following: (I) R0 resection could not be achieved, or the presence of distant metastatic lesions; (II) history of other malignant tumors and/or upper gastrointestinal surgery; (III) patients who had received previous chemotherapy before enrollment. Primary outcome measures were to calculate the total number of lymph nodes detected and the number of metastatic lymph nodes. The secondary endpoints: lymph node staining rate, lymph node metastasis rate, and the number of micro lymph nodes detected, which was aimed to reflect the degree of difficulty of picking up lymph nodes. Figure 1 shows the trial scheme. This study was approved by the Ethics Committee of the Fourth Hospital of Hebei Medical University, and informed consent was obtained from each patient. The ethics approval document number was 2018009, and the Chinese Clinical Trial Registration number was ChiCTR2100047407. All procedures performed in this study involving human participants were in accordance with the Declaration of Helsinki (as revised in 2013).

\section{Carbon nanoparticles}

Carbon nanoparticles were applied in the form of injection of a standard suspension $(1 \mathrm{~mL} ; 50 \mathrm{mg})$, with a diameter of $150 \mathrm{~nm}$. As the capillary endothelium 


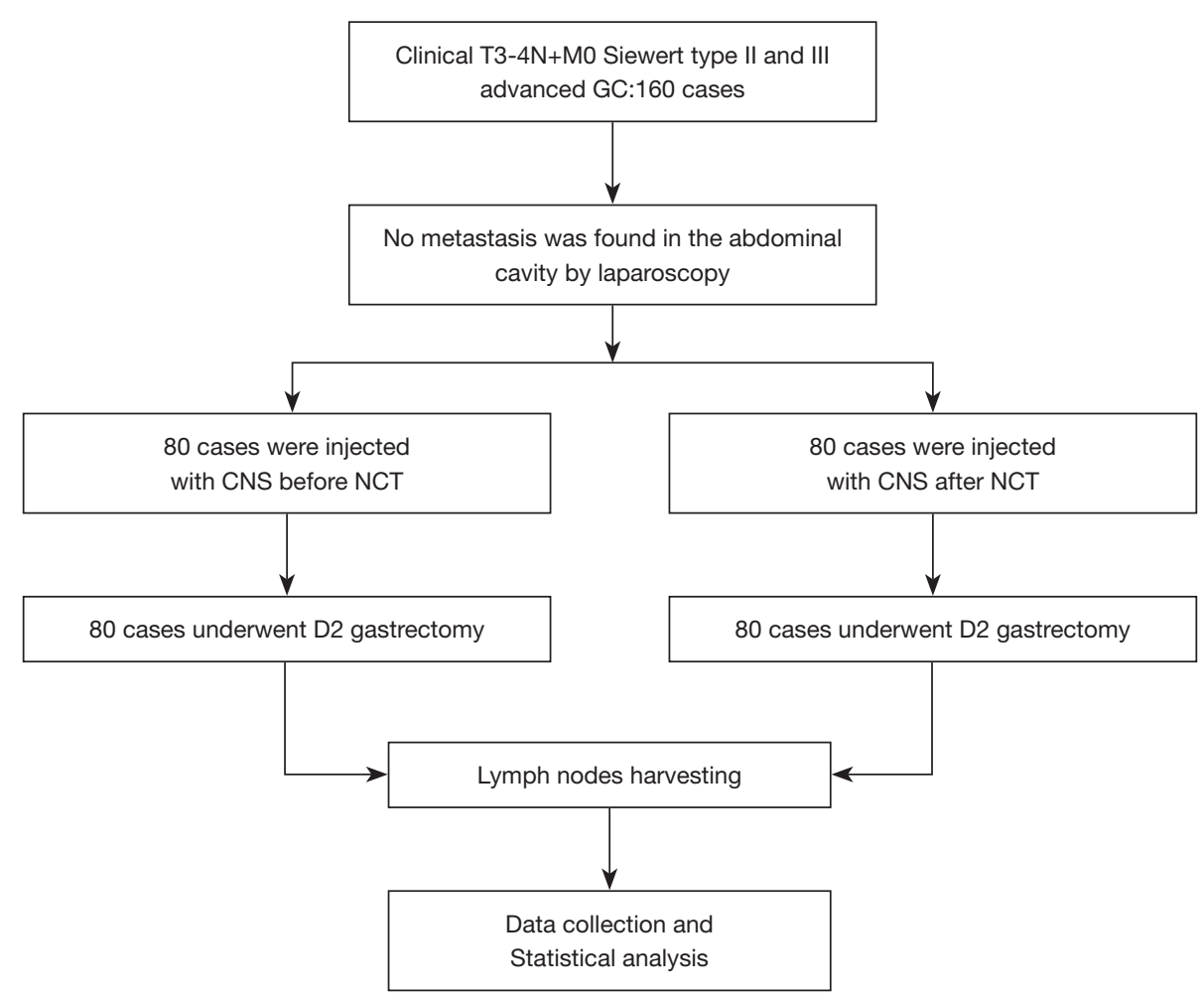

Figure 1 Trial scheme. CNS, carbon nanoparticles suspension injections; NCT, neoadjuvant chemotherapy; GC, gastric cancer.

contains 20-50 nm wide pores and capillary lymphatic cells contain pores $100-500 \mathrm{~nm}$ wide, nano-carbon does not enter the blood vessel but instead, rapidly enters the draining lymphatic vessels and lymph nodes. Consequently, the lymph nodes retain a large amount of ultra-fine carbon particles dyeing them black and facilitating their identification during surgery.

\section{Endoscopic injection of CNS}

Nano-carbon was injected at a distance of $0.5-1 \mathrm{~cm}$ from the edge of the tumor into four sites: the submucosa in the cardia side, the pylorus side, the large curved side, and the small curved side, with $0.25 \mathrm{~mL}$ at each point (Figure $2 A$ ). This operation was performed by a specialist.

\section{CNS injection timing selection}

The experimental group received endoscopic injection of CNS within 24 hours prior to NCT, while the control group received this post NCT and within 24 hours before D2 radical resection. SOX [oxaliplatin: $130 \mathrm{mg} /$ (body surface area, BSA): $\mathrm{m}^{2}$, first day $+\mathrm{S}-1$ : (BSA: $<1.25 \mathrm{~m}^{2}$,
$40 \mathrm{mg}$ each time; $\geq 1.25$ to $<1.5 \mathrm{~m}^{2}, 50 \mathrm{mg}$ each time; $\geq 1.5 \mathrm{~m}^{2}, 60 \mathrm{mg}$ each time), 2 times a day, for 2 weeks] was chosen as the NCT regimen, each cycle taking three weeks, and each patient receiving four cycles before open or laparoscopic D2 radical surgery.

\section{Intraoperative lymph node dissection range and postoperative pathology}

After four cycles of NCT all patients were treated with open or laparoscopic D2 radical gastrectomy, and lymph node classification was based on the 15 th edition of the Japanese Gastric Cancer Regulations (10). As carbon nanoparticles injected under the gastric mucosa can enter lymphatic vessels and lymph nodes, those draining from the tumor region were stained black allowing easy identification during surgery (Figure $2 B$ and Figure 3). After the specimen was isolated, the nodes were examined by trained physicians, and their total number and staining rate were counted, while micro lymph nodes were measured with vernier calipers and counted (Figure 3). According to the postoperative pathological results, the lymph node metastasis rate and pathological $\mathrm{N}$ stage of each group were 

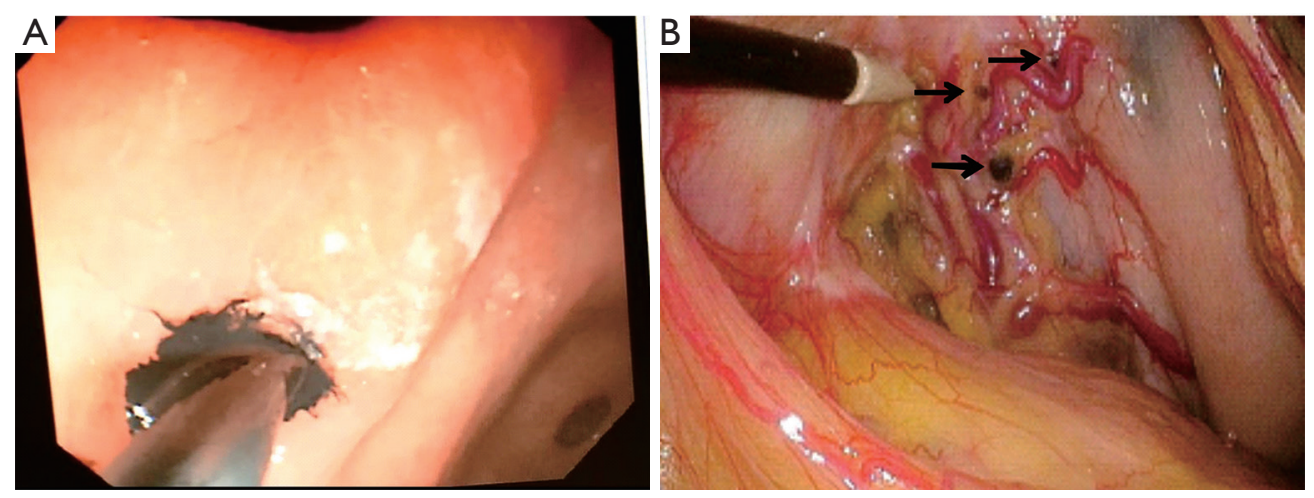

Figure 2 CNS was injected under the gastric mucosa by endoscopy, and black lymph nodes were seen intraoperatively. (A) Carbon nanoparticles were injected under the gastric mucosa under endoscopy; (B) black lymph nodes (black arrows) were seen during the operation. CNS, carbon nanoparticles suspension injections.
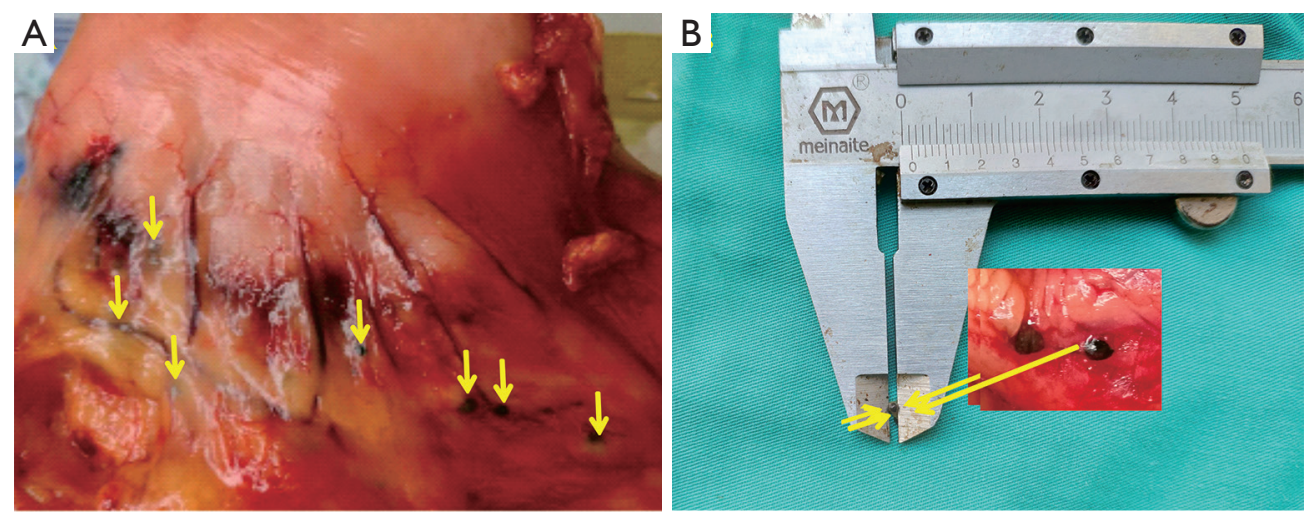

Figure 3 The diameter of the black lymph nodes in the surgical specimen was measured with a vernier caliper. (A) Black lymph nodes on surgical specimen (yellow array); (B) black micro lymph nodes $(\mathrm{d}<2 \mathrm{~mm}$ ) measured with a vernier caliper (yellow array).

also counted.

\section{Surgical complications}

The incidence of postoperative complications, including pulmonary infection, incision infection, bleeding, anastomotic fistula, and intestinal obstruction, were compared in each group.

\section{Statistical analysis}

SPSS 22.0 statistical software was also used for data analysis. Measurement data were expressed as mean \pm standard deviation, compared by $t$-test and analysis of variance. Enumeration data were expressed as percentage using the $\chi^{2}$ test, while the grade data were tested by nonparametric rank sum test. $\mathrm{P}<0.05$ was regarded as statistically significance.

\section{Results}

\section{Patient demographics and cancer characteristics}

One hundred and sixty patients diagnosed with advanced GC who planned to receive NCT from January 2020 to January 2021 were recruited, of the 160 enrolled patients there were 54 males and 26 females in the experimental group, 51 males and 29 females in the control group. All enrolled patients received D2 radical resection. The database of this study was established based on the following information: age, gender, body mass index (BMI), tumor location, tumor longest diameter, clinical TNM stage, Luaren classification, tumor regression grade (TRG), differentiation degree, surgery range, total 
Table 1 Comparison of baseline data between the two groups

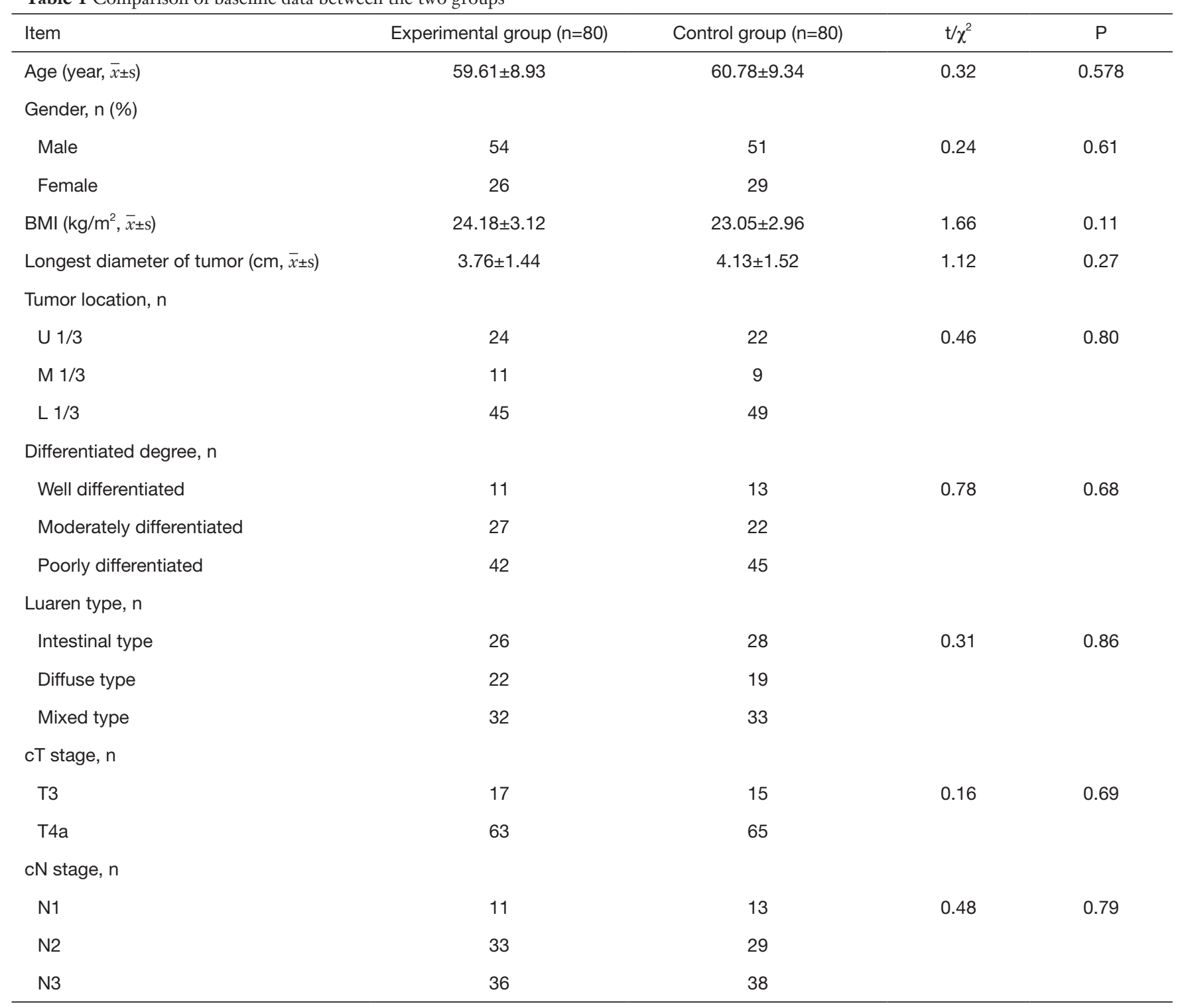

BMI, body mass index.

number of lymph nodes detected, the number of stained lymph nodes, the number of lymph node metastases, the number of stained lymph node metastases, the number of micro lymph nodes (diameter $<2 \mathrm{~mm}$ ) detected, and postoperative complications including pulmonary infection, wound infection, anastomotic leakage, and intestinal obstruction. There was no significant difference between the two groups in terms of age, sex, BMI, tumor size, tumor location, Luaren classification, clinical TNM staging, and differentiation degree (all $\mathrm{P}>0.05$ ) (Table 1, Figure 1).

\section{Operation and lymph node detection between the two groups}

In the experimental group 35 patients underwent total gastrectomy and 45 underwent distal gastrectomy, while in the control group 32 underwent total gastrectomy and 48 underwent distal gastrectomy. Analysis showed there was no significant difference between the two groups. Postoperative pathological TRG was also not significantly different between the two groups. A total of 3,197 lymph nodes were detected in the experimental group, including 384 metastatic lymph nodes, 1,424 stained lymph nodes, 
Table 2 Comparison of operative and postoperative pathological data between the two groups

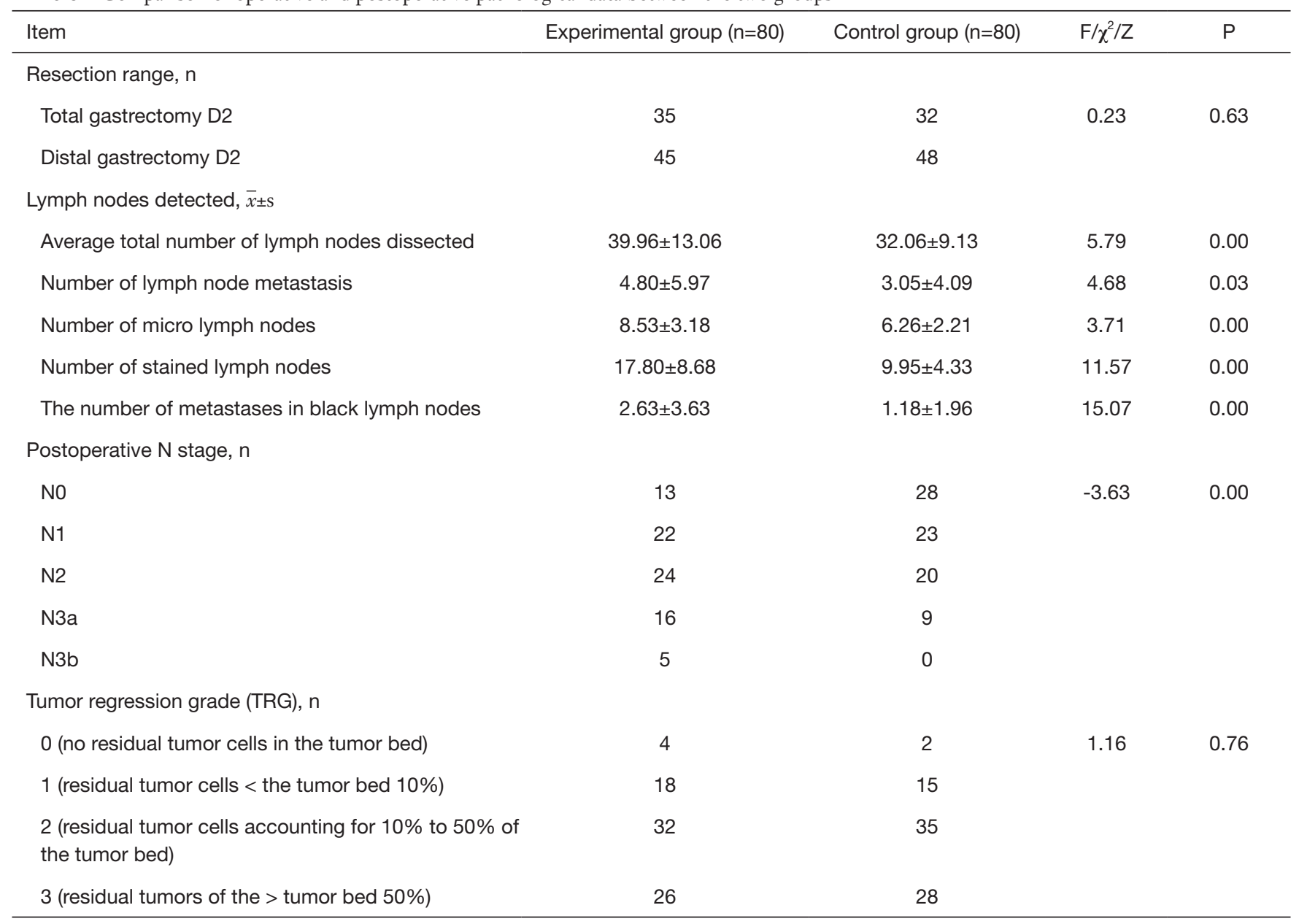

and 210 metastatic stained lymph nodes. The total number of lymph nodes in the control group was 2,565, including 244 metastatic lymph nodes, 796 stained lymph nodes, and 94 metastatic stained lymph nodes. The number of lymph nodes, the number of metastatic lymph nodes, the number of small lymph nodes, the rate of lymph node staining, and the rate of lymph node metastasis were all higher in the experimental group than in the control group (all $\mathrm{P}>0.05$ ) (Table 2).

\section{Rate of lymph node staining compared between the two groups}

The number of stained and unstained lymph nodes in the experimental group was 1,424 and 1,773 , respectively, with a staining rate of $44.54 \%(1,424 / 3,197)$. In the control group, the number of stained and unstained lymph nodes was 796 and 1,769 , respectively, with a staining rate of $31.03 \%(796 / 2,565)$. The staining rate of the experimental group was higher than that of the control group, and the difference was statistically significant $(\mathrm{P}<0.05)$ (Table 3).

\section{Rate of lymph node metastasis between the two groups}

The number of lymph node metastases in the experimental group was 384 , and the number of lymph node metastases with black staining was 210 . The lymph node metastasis rate was $12.01 \%(384 / 3,197)$ and the black staining lymph node metastasis rate was $14.75 \%(210 / 1,424)$. In the control group, the number of lymph node metastases was 244 , the number of lymph node metastases with black staining was 94 , the rate of lymph node metastasis was $9.51 \%$ $(244 / 2,565)$, and the rate of lymph node metastasis stained was $11.81 \%$ (94/796). The lymph node metastasis rate of 
Table 3 Rates of lymph node staining and metastasis compared between the two groups

\begin{tabular}{lcccc}
\hline Item & Experimental group & Control group & $\chi^{2}$ & $\mathrm{P}$ \\
\hline \multicolumn{2}{l}{ Lymph nodes stained } & & & \\
Yes & 1,424 & 796 & & \\
No & 1,773 & 1,769 & & \\
Lymph node metastasis & & & \\
Yes & 384 & 244 & & \\
No & 2,813 & 2,321 & & 0.000 \\
Stained lymph node metastasis & & & \\
Yes & 210 & 94 & & \\
No & 1,214 & 702 & 3.73 & 0.053 \\
\hline
\end{tabular}

the experimental group was higher than that of the control group $(\mathrm{P}<0.05)$ (Table 3).

\section{Postoperative pathological $N$ staging of the two groups}

In the experimental group 13 patients were staged as N0, 22 as $\mathrm{N} 1,24$ as N2, 16 as N3a, and 5 as N3b, while the control group saw 28 as $\mathrm{N} 0,23$ as N1, 20 as N2, 9 as N3a and 0 as N3b. More metastatic lymph nodes were detected in the experimental group than that in the control group, and postoperative $\mathrm{N}$ staging was more accurate in the experimental group, with significant difference between the two $(\mathrm{P}<0.05)$ (Table 2).

\section{Incidence of postoperative complications}

There were five cases of postoperative pulmonary infection and two of anastomotic fistula in the experimental group, and no bleeding, incision infection, intestinal obstruction or other complications. In the control group there was one case of postoperative bleeding, six of pulmonary infection, one of incision infection, one of anastomotic fistula, and no intestinal obstruction and other complications. No statistically significant differences in complications between the two groups was seen $\left(\chi^{2}=0.28, P>0.05\right)$.

\section{Discussion}

Several clinical trials in recent years have demonstrated NCT can improve the radical cure rate and 5-year survival rate after surgery of GC including the MAGIC and JCOG
0405 trials (5-7). While this has resulted in NCT for GC being written into the NCCN guidelines, as Lordick (11) contends, NCT may also make tumor tissue and regional lymph nodes shrink, making their detection more difficult during operation and affecting the accuracy of $\mathrm{N}$ staging. Although NCCN guidelines emphasize the accuracy of N staging and require at least 16 lymph nodes to be detected in each radical resection of GC specimens (12), studies have confirmed that in advanced GC, the number of potentially positive lymph nodes can only be increased when more than 30 lymph nodes are submitted for examination, thereby reducing the $\mathrm{N}$ staging offset and ensuring the accuracy of $\mathrm{N}$ staging $(2,3)$.

Lymph node detection methods include macroscopic examination, finger palpation, and lymph node tracer methods $(13,14)$. CNS is a suspension made of polyvinylpyrrolidone and normal saline, wrapped in smooth carbon particles, with an average diameter of $150 \mathrm{~nm}$. As the intercellular space of capillary endothelial cells is $20-50 \mathrm{~nm}$, and that of capillary lymphatic cells is $100-500 \mathrm{~nm}$, CNS only enters lymphatic capillaries; $1.0 \mathrm{~mL}$ of CNS was drawn into a $1 \mathrm{ml}$ syringe and injected into the submucosa of the stomach at four points around the tumor $2 \mathrm{~cm}$ away on average, each point was injected with $0.25 \mathrm{~mL}$. After the injection, each injection point was lightly pressed with dry gauze for 10 seconds to prevent the tracer extravasation from affecting the staining effect. From here it can rapidly enter lymphatic vessels to be retained in lymph nodes through macrophage phagocytosis, and can be retained in both for periods of 3-4 months providing an excellent tracer effect (15). In addition, CNS has no biological activity, is non-toxic, and will not cause adverse reactions (16). However, as NCT can cause lymphatic contraction and fibrosis in surrounding tumor tissue, CNS injection after NCT often affects the effective detection of intraoperative lymph nodes due to the decreased degree of lymphatic staining $(17,18)$. Labeling CNS before NCT can theoretically provide a long enough dispersion time and avoid the influence of lymph node tracer tracing caused by NCT on lymph node contraction and fibrosis in surrounding tumor tissues $(15,17,18)$. Therefore, in patients with advanced GC, providing NCT before injection facilitates the diffusion of CNS within lymph nodes, and can, theoretically, improve their staining, reduce the effect of NCT, and increase the detection of smaller and greater numbers of nodes to improve the precision of postoperative $\mathrm{N}$ staging. In this study, compared with the application of CNS before NCT, the staining rate and metastasis rate of lymph nodes detected were significantly increased, and the injection of carbon nanoparticles before NCT improved the number 
of lymph nodes detected without increasing postoperative complications. Among the black-stained lymph nodes detected, the number of black-stained lymph nodes in the experimental group was higher than that in the control group, but there was no statistical difference. The reason may be related to the limited number of enrolled cases. With the increase of the number of enrolled cases, there may be significant statistical difference. There were significant differences in postoperative pathological $\mathrm{N}$ staging between the two groups, indicating the application of CNS before NCT could significantly increase the number of lymph nodes and metastasis detected in a safe and effective manner, and render the postoperative pathological lymph node staging more accurate, indicating the use of carbon nanoparticles was safe and effective. At present, domestic and foreign scholars define lymph nodes with diameter less than $2 \mathrm{~mm}$ as micro lymph nodes, and some studies have found that the metastasis rate of these nodes can exceed $17 \%$ of the total number of positive nodes (19). This study showed that the detection rate of small lymph nodes in the experimental group was significantly higher than in the control group, indicating CNS could increase the number of micro lymph nodes detected, and the tracer effect of CNS injection before NCT was better.

In conclusion, the injection of CNS before NCT can increase the total number of lymph nodes and number of metastatic lymph nodes detected, improve the accuracy of $\mathrm{N}$ staging after NCT for GC, and create a more accurate estimation of the postoperative pathological staging and prognosis of patients. Moreover, it can improve follow-up treatment and evaluate the prognosis of patients. However, long-term follow-up and observation are required to verify the results and improve the survival of patients.

\section{Acknowledgments}

Funding: This study was funded by the Hebei Medical Science Research project, No. 20211338; Hebei Medical Application Tracking project, No. 2703006; Hebei Health Commission County-level Public Hospital Appropriate Health Technology Promotion project, No. 2019024; and Hebei Government-funded Clinical Medicine Outstanding Talent Training project, No. 2019012.

\section{Footnote}

Reporting Checklist: The authors have completed the CONSORT reporting checklist. Available at https://dx.doi. org/10.21037/jgo-21-457
Trial Protocol: Available at https://dx.doi.org/10.21037/jgo$21-457$

Data Sharing Statement: Available at https://dx.doi. org/10.21037/jgo-21-457

Conflicts of Interest: All authors have completed the ICMJE uniform disclosure form (available at https://dx.doi. org/10.21037/jgo-21-457). All authors reported that this study was funded by the Hebei Medical Science Research project, No. 20211338; Hebei Medical Application Tracking project, No. 2703006; Hebei Health Commission Countylevel Public Hospital Appropriate Health Technology Promotion project, No. 2019024; and Hebei Governmentfunded Clinical Medicine Outstanding Talent Training project, No. 2019012. The authors have no other conflicts of interest to declare.

Ethical Statement: The authors are accountable for all aspects of the work in ensuring that questions related to the accuracy or integrity of any part of the work are appropriately investigated and resolved. All procedures performed in this study involving human participants were in accordance with the Declaration of Helsinki (as revised in 2013). This study was approved by the Ethics Committee of the Fourth Hospital of Hebei Medical University, and informed consent was obtained from each patient. The ethics approval document number was 2018009 , and the Chinese Clinical Trial Registration number was ChiCTR2100047407.

Open Access Statement: This is an Open Access article distributed in accordance with the Creative Commons Attribution-NonCommercial-NoDerivs 4.0 International License (CC BY-NC-ND 4.0), which permits the noncommercial replication and distribution of the article with the strict proviso that no changes or edits are made and the original work is properly cited (including links to both the formal publication through the relevant DOI and the license). See: https://creativecommons.org/licenses/by-nc-nd/4.0/.

\section{References}

1. Ajani JA, Bentrem DJ, Besh S, et al. Gastric cancer, version 2.2013: featured updates to the NCCN Guidelines. J Natl Compr Canc Netw 2013;11:531-46.

2. Sano T, Coit DG, Kim HH, et al. Proposal of a new stage grouping of gastric cancer for TNM classification: 
International Gastric Cancer Association staging project. Gastric Cancer 2017;20:217-25.

3. Deng J, Liu J, Wang W, et al. Validation of clinical significance of examined lymph node count for accurate prognostic evaluation of gastric cancer for the eighth edition of the American Joint Committee on Cancer(AJCC) TNM staging system. Chinese Journal of Cancer Research 2018;30:477-91.

4. Ajani JA, D'Amico TA, Almhanna K, et al. Gastric Cancer, Version 3.2016, NCCN Clinical Practice Guidelines in Oncology. J Natl Compr Canc Netw 2016;14:1286-312.

5. Cunningham D, Allum WH, Stenning SP, et al. Perioperative chemotherapy versus surgery alone for resectable gastroesophageal cancer. $\mathrm{N}$ Engl J Med 2006;355:11-20.

6. Ychou M, Boige V, Pignon JP, et al. Perioperative chemotherapy compared with surgery alone for resectable gastroesophageal adenocarcinoma: an FNCLCC and FFCD multicenter phase III trial. J Clin Oncol 2011;29:1715-21.

7. Tsuburaya A, Mizusawa J, Tanaka Y, et al. Neoadjuvant chemotherapy with S-1 and cisplatin followed by D2 gastrectomy with para-aortic lymph node dissection for gastric cancer with extensive lymph node metastasis. Br J Surg 2014;101:653-60.

8. Wu ZM, Teng RY, Shen JG, et al. Reduced lymph node harvest after neoadjuvant chemotherapy in gastric cancer. J Int Med Res 2011;39:2086-95.

9. Hsieh FJ, Wang YC, Hsu JT, et al. Clinicopathological features and prognostic factors of gastric cancer patients aged 40 years or younger. J Surg Oncol 2012;105:304-9.

10. The Japanese Society for Cancer of the Stomach. Gastric cancer treatment protocol. Tokyo: Gold Original

Cite this article as: Yang P, Tian Y, Tan B, Ding P, Guo H, Liu Y, Zhang Z, Li Y, Zhao Q. Clinical application of nano-carbon to improve the accuracy of lymph node staging in patients with advanced gastric cancer receiving neoadjuvant chemotherapy: a prospective randomized controlled trial. J Gastrointest Oncol 2021;12(5):2052-2060. doi: 10.21037/jgo-21-457
Publishing Corporation, 2017.

11. Lordick F, Ott K, Sendler A. Gastric cancer and adenocarcinoma of the esophagogastric junction: principles of neoadjuvant therapy. Chirurg 2011;82:968-73.

12. In H, Solsky I, Palis B, et al. Validation of the 8th Edition of the AJCC TNM Staging System for Gastric Cancer using the National Cancer Database. Ann Surg Oncol 2017;24:3683-91.

13. Aoyama T, Fujikawa H, Cho H, et al. A methylene blueassisted technique for harvesting lymph nodes after radical surgery for gastric cancer: a prospective, randomized, controlled study. Am J Surg Pathol 2015;39:266-73.

14. Li Z, Ao S, Bu Z, et al. Clinical study of harvesting lymph nodes with carbon nanoparticles in advanced gastric cancer: a prospective randomized trial. World J Surg Oncol 2016;14:88.

15. Lucci A, Turner RR, Morton DL. Carbon dye as an adjunct to isosulfan blue dye for sentinel lymph node dissection. Surgery 1999;126:48-53.

16. Magrez A, Kasas S, Salicio V, et al. Cellular toxicity of carbon-based nanomaterials. Nano Lett 2006;6:1121-5.

17. Yokota T, Saito T, Narushima Y, et al. Lymph-node staining with activated carbon CH40: a new method for axillary lymph-node dissection in breast cancer. Can J Surg 2000;43:191-6.

18. Schwarz RE, Smith DD. Clinical impact of lymphadenectomy extent in resectable gastric cancer of advanced stage. Ann Surg Oncol 2007;14:317-28.

19. Baxter NN, Virnig DJ, Rothenberger DA, et al. Lymph node evaluation in colorectal cancer patients: a populationbased study. J Natl Cancer Inst 2005;97:219-25.

(English Language Editor: B. Draper) 